BULLETIN Bulletin hispanique

HISPANIQUE Université Michel de Montaigne Bordeaux

119-1 | 2017

Autorité et pouvoir dans le théâtre du Siècle d'Or

\title{
Du pouvoir temporel à l'autorité de l'amour divin dans Doña Beatriz de Silva de Tirso de Molina
}

Isabelle Bouchiba-Fochesato

\section{(2) OpenEdition}

Édition électronique

URL : http://journals.openedition.org/bulletinhispanique/4763

DOI : 10.4000/bulletinhispanique.4763

ISBN : 979-10-300-0142-6

ISSN : $1775-3821$

Éditeur

Presses universitaires de Bordeaux

Édition imprimée

Date de publication : 15 juin 2017

Pagination : $59-78$

ISBN : 979-10-300-0141-9

ISSN : 0007-4640

\section{Référence électronique}

Isabelle Bouchiba-Fochesato, «Du pouvoir temporel à l'autorité de l'amour divin dans Doña Beatriz de Silva de Tirso de Molina », Bulletin hispanique [En ligne], 119-1 | 2017, mis en ligne le 15 juin 2020, consulté le 11 septembre 2020. URL : http://journals.openedition.org/bulletinhispanique/4763 ; DOI https://doi.org/10.4000/bulletinhispanique.4763 


\title{
Du pouvoir temporel à l'autorité de l'amour divin dans Doña Beatriz de Silva de Tirso de Molina
}

\author{
IsABELLE Bouchiba-Fochesato \\ Université Bordeaux Montaigne - Ameriber
}

La pièce Doña Beatriz de Silva met en scène l'histoire du personnage éponyme connu pour avoir reçu, de la Vierge elle-même, la révélation de sa conception immaculée. L'article se propose d'analyser le rôle central du personnage de Beatriz et de l'amour qu'elle inspire dans le cadre d'une théorie du pouvoir qui ne serait justifié que par l'autorité de l'amour.

Mots-clés : Tirso de Molina, autorité, pouvoir, théâtre, Doña Beatriz de Silva.

La obra Doña Beatriz de Silva pone en escena la historia del personaje epónimo conocido por haber recibido de la Virgen la revelación de su inmaculada concepción. El artículo se propone analizar el papel central del personaje de Beatriz y del amor que inspira en el ámbito de una teoría del poder que solo el amor divino podría justificar.

Palabras claves: Tirso de Molina, autoridad, poder, teatro, Doña Beatriz de Silva.

"Doña Beatriz de Silva" tells the story of the eponymous character known to have received from the Virgin herself the revelation of her immaculate conception. The article proposes an analyse of the central role of Beatriz's character and the love she inspires in the framework of a theory of power that would be justified only by the authority of love.

Keywords: Tirso de Molina, authority, power, theater, Doña Beatriz de Silva. 
T a pièce Doña Beatriz de Silva a semble-t-il été écrite par Tirso de Molina Lentre 1617 et $1621^{1}$. Son action se déroule pour l'essentiel à la cour du roi Juan II et de son épouse, la reine Isabel du Portugal, à la fin du XVe siècle. À la suite d'un malheureux échange de portraits, le roi Juan s'attend à découvrir sa nouvelle épouse, Isabel, sous les traits de la suivante de cette dernière, Beatriz. Le roi n'étant pas parvenu à dissimuler sa méprise (il s'adresse à Beatriz en lieu et place d'Isabel lors de leur première rencontre) la reine est immédiatement en proie à une terrible jalousie. Une fois installée à la cour de Castille, Beatriz subjugue tous les galants présents, y compris le roi lui-même (sans que ce dernier souhaite pour autant faire disparaître son épouse), au point que la reine Isabel tente d'assassiner la dame après avoir obtenu la preuve de la passion du roi pour elle. Beatriz est miraculeusement sauvée d'une mort horrible (elle a été enfermée dans une armoire sans eau ni nourriture et manque y périr au bout de trois jours de captivité) par la Vierge Marie, qui lui apparaît sous les traits d'une fillette. La dame s'enfuit ensuite de la cour guidée par saint Antoine, pour le monastère de Santo Domingo el Real de Tolède afin d'y fonder un ordre marial voué au culte de l'Immaculée Conception. Elle est néanmoins rattrapée par le roi et la reine, revenus de leurs égarements respectifs, lesquels lui promettent de la soutenir dans son entreprise.

Parallèlement à cette intrigue principale, s'en déroule une seconde, puisque l'un des frères de Beatriz, lui aussi nommé Juan, épris depuis sa jeunesse de la belle Leonor avec qui il a été élevé, croit déceler chez elle un sentiment similaire lorsqu'elle le convie à l'accompagner en Allemagne en tant que gentilhomme, pays où elle se rend pour épouser l'empereur Federico III. Comprenant cependant après quelque temps d'une cour assidue que l'amour qui anime Leonor est un sentiment purement fraternel, Juan décide d'entrer dans les ordres et de se consacrer à la règle de Saint François.

Mêlant tout au long des trois actes des traits de la comedia "politique» ou historique (le début de l'acte I développe une courte trame autour de la figure de don Álvaro de Luna, lequel a arrangé le mariage de Juan II et d'Isabel) et de la comedia palaciega ${ }^{2}$ aux multiples intrigues amoureuses, la pièce s'oriente donc finalement au troisième acte vers la comedia hagiographique ${ }^{3}$.

Qu'il s'agisse d'une ouvre de commande ${ }^{4}$ ou non, Doña Beatriz est généralement considérée comme une pièce visant à promouvoir le culte marial et plus précisément le culte de l'Immaculée Conception ${ }^{5}$ à une époque de

1. Luis Vázquez estime que la pièce a été rédigée en 1618 dans son artice intitulé « Editar la prosa de Tirso de Molina ", Alicante : Biblioteca Virtual Miguel de Cervantes, 2006. Quoi qu'il en soit, la pièce fait partie des 53 œuvres authentifiées par Blanca Oteiza dans son article ¿Conocemos los textos verdaderos de Tirso de Molina? p. 111.

2. Pour María del Pilar Palomo Vázquez la pièce met en scène l'histoire de "la fundadora de las Concepcionistas, con fuertes elementos históricos, aunque con derivación, en muchas ocasiones, a la comedia palaciega " in La creación dramática de Tirso de Molina (II) http://www.ucm.es/OTROS/ especulo/numero8/palomo2.htm.

3. Maurel $1971: 357-359$.

4. Florit 2007-2008: 441-450, 446

5. Manuel Tudela, dans l'introduction très complète qu'il consacre à la pièce éditée à l'IET 
controverse théologique entre immaculistes et maculistes - et de victoires doctrinales des premiers sur les seconds ${ }^{6}$ - tout en veillant à inscrire la royauté espagnole au cœur même du système des valeurs chrétiennes post-tridentines et à légitimer le pouvoir royal espagnol comme émanation de l'autorité divine 7 . La pièce met en effet en scène la révélation, par la Vierge elle-même, à Beatriz de Silva - dame de Cour de la reine Isabel, ainsi que nous l'avons dit - de sa conception sans péché ainsi que la soumission du roi Juan II de Castille et de son épouse Isabel à la volonté mariale. De la même manière, la pièce est également souvent taxée de thématiquement hétérogène dans la mesure où elle semble mêler histoire, politique, intrigues palatines et amoureuses et hagiographie et ce pour mieux capter l'attention d'un public exigeant et indiscipliné.

Si tous ces éléments sont bien évidemment avérés, ils nous semblent tous relever d'une réception relativement superficielle tant de l'intrigue à proprement parler que de l'appareil dramatique et poétique mis en jeu pour lui donner vie : en conséquence, ils pourraient masquer le sens profond de l'œuvre.

La pièce en effet, ainsi que nous allons le montrer dans les pages qui suivent, élabore une réflexion subtile et remarquablement profonde sur le pouvoir et l'autorité en mettant en scène un renversement dialectique entre ces deux notions à partir d'un élément transversal qui dépasse, grandit et transcende le destin de chacun des personnages : l'amour. Manuel Tudela, dans l'introduction qu'il consacre à la pièce, introduction déjà citée en note, ne manque pas de relever l'importance du thème de l'amour comme élément dramatique structurant :

Dramáticamente, la importancia del amor es capital. Si antes hemos considerado a la Inmaculada como impulso último de la obra, el amor es por otro lado el motor dramático de la comedia en el sentido en que se convierte en el factor que lanza a los personajes al movimiento ${ }^{8}$.

écrit (entre autres) "El diálogo entre Beatriz y la Virgen constituye una auténtica declaración inmaculista [...]", p. 851 et plus loin "La postura de Tirso es inequívoca : imbuido en el sentir de su época, en el carisma de la Orden mercedaria y, probablemente, por convicción personal, defiende la tesis inmaculista ajustándose a las teorías teológicas que la sostenían ", Tirso de Molina 1999 : 852.

6. Si le culte de l'Immaculée Conception n'est solennellement fixé comme dogme qu'au Concile de Vatican I le 8 décembre 1854 (notamment à la suite d'apparitions de la Vierge dans une chapelle de la rue du Bac à Paris à une jeune novice parisienne, Catherine Labouré) la controverse théologique remonte aux Pères de l'Église et agite tout le Moyen Âge, les maculistes étant plutôt du côté des dominicains et les immaculistes du côté des franciscains. Le Concile de Trente affirme la conception sans péché de la mère du Christ mais sans l'ériger cependant en dogme, Paul V en 1617 autorise l'enseignement de l'Immaculée conception (et interdit d'exprimer publiquement - mais non en privé - une opinion contraire) et l'époque baroque foisonne en œuvres artistiques (picturales essentiellement) promouvant cette croyance (Tirso lui-même passe trois ans à Saint-Domingue dans ce but).

7. Manuel Tudela estime ainsi que "La intervención de la divinidad y la decisión de Beatriz contribuyen a poner un poco de orden en un palacio notablemente alterado. En este sentido, la comedia participa de la visión optimista sobre el poder que Tirso imprime a la mayoría de sus comedias de carácter politico : el desorden en el sistema de gobierno es remediado satisfactoriamente cunado los que ostentan el poder se deciden a utilizarlo con rectitud, y ahi acaba el asunto ".Tirso de Molina 1999 : p. 856

8. Ibidem, p. 853 
Il nous semble cependant que dans cette analyse subsiste une dichotomie assez marquée entre amour et pouvoir ainsi qu'une sous-évaluation de la question du pouvoir lui-même (et de l'autorité qui le fonde). Cette sousévaluation apparait, précisément, dans le fait de dissocier les deux thèmes de l'amour et du pouvoir lors de leur présentation dans l'introduction que nous citons ici. L'action de Beatriz et celle de la Vierge y sont certes décrites comme capables d'apaiser les tensions que connait la cour mais ceci apparaît comme une sorte d'épiphénomène opérant de manière assez mécanique ainsi que le montre le commentaire de Manuel Tudela quant aux actes du roi : "Se apunta pues, como también en el personaje de Isabel, un leve tratamieno de las cuestiones de gobierno que se centra en la crítica hacia la utilización incorrecta del poder. La intervención de la divinidad y la decisión de Beatriz contribuyen a poner un poco de orden en un palacio notablemente alterado $"{ }^{9}$. Or le véritable défi de la pièce tirsienne consiste, nous semble-t-il, à révéler la nature profonde de la relation entre pouvoir humain et autorité de l'amour divin par le truchement d'un personnage christique qui, pour reprendre les mots de Françoise Dolto, tel Jésus [...] enseigne le désir et y entraîne ${ }^{10}$.

Dans le but d'essayer de démontrer la validité de cette lecture de l'œuvre, nous nous attacherons, dans un premier temps, aux manifestations de pouvoir temporel qui émaillent la pièce et au cas particulier de don Juan de Meneses, premier personnage à subir un grave revers amoureux et pièce importante de l'architecture de la pièce. Puis, dans un second temps, nous observerons les effets produits par la seule présence de doña Beatriz sur les galants mais aussi, et surtout, sur les figures de pouvoir que sont don Juan II et doña Isabel de Portugal. Enfin, nous nous attacherons à mettre en relief la nature profonde du personnage de Beatriz et à en montrer le rôle déterminant à l'heure de convoquer les notions de pouvoir et d'autorité.

Le pouvoir temporel (et ses représentations symboliques et concrètes) est sans aucun doute le tout premier élément à s'imposer au lecteur-spectateur dès le début de la pièce. La didascalie initiale fait ainsi état d'une exhibition remarquable des marques extérieures de pouvoir : Tiros de artilleria : música de todo género, fiestas dentro, y saca Silveira sobre los corredores de arriba, a un lado, una bandera con las armas de Portugal y Castilla et quelques vers plus loin, une seconde didascalie nous montre un second déploiement d'étendards : Disparan $y$ tocan chirimias. [...] Al otro lado saca arriba Olivenza otra bandera con las armas de Portugal y del Imperio.

Ces festivités royales célèbrent, on le sait, le double mariage d'Isabel du Portugal et de Juan II de Castille d'une part et de l'Infante Leonor avec l'empereur Allemand Federico III d'autre part. Lorsque la pièce commence,

9. Opus cité, p. 855.

10. Dolto $1977: 15$ Cette phrase de la psychanaliste Françoise Dolto a, à nos yeux, le mérite d'offrir une formulation contemporaine et d'une remarquable densité, de la séduction non profane mais divine exercée par le Christ et transposée ici au personnage de dońa Beatriz. Elle synthétise en quelque sorte ce que la poésie sacrée, à commencer par le Cantique des cantiques, n’a cessé de dire depuis longtemps. 
une future reine se sépare donc d'une future impératrice. Sont présents dans le discours et/ou sur scène le roi don Juan de Castille, l'empereur Federico III d'Allemagne mais aussi l'infant don Enrique, frère du roi castillan. Le discours des premières scènes de la pièce est rapidement saturé de signifiants se rapportant au paradigme de la royauté et du pouvoir (reino, reina, emperatriz, rey, alteza, infanta, imperio, corte, guerra, paz etc.). Cette saturation culmine lors des adieux entre les deux amies appelées à régner loin de leur Lisbonne natale :

Leonor: Adiós, reina de Castilla.

Isabel: Augusta alemana, adios ${ }^{11}$.

Parallèlement à ce paradigme qui perdure tout au long de la pièce, quoique en mode mineur, dès le début de l'œuvre s'y déroulent un second puis un troisième qui vont, avec le premier, constituer le nœud conceptuel de la pièce. De fait, simultanément à la convocation du pouvoir royal dans la toute première adresse de la pièce, surgit celui de la beauté (le premier mot de la pièce est l'adjectif hermosa $a^{12}$ pour qualifier doña Isabel), puis, très vite celui de l'amour et de la jalousie. De fait, immédiatement après la scène triomphale qui ouvre la pièce, apparaissent don Juan de Meneses, un des frères de doña Beatriz, et son ami don Fernando. Don Juan fait part à son compagnon du désespoir dans lequel le plongent le départ et le mariage de Leonor avec qui il a été élevé et dont il est éperdument amoureux. En dépit de tous les appels à la raison de don Fernando, don Juan refuse de se plier au principe de réalité qu'invoque son ami allant jusqu'à se transformer en une Didon au masculin dans une adresse à la tonalité de fin du monde :

\author{
Juan :Ya se parte de Lisboa; \\ ya, conde, se va a embarcar \\ sobre los hombros del Tajo, \\ que de perlas y coral \\ guarneciendo su cabeza, \\ celos tiene porque el mar \\ en sus brazos la reciba, \\ y su azul hurtando está, \\ como yo que, imagen suya \\ de los muros de San Gián, \\ arrojándome a sus olas \\ mi fuego he de sepultar, \\ pues en mortajas turquies \\ bien los celos morirán, \\ que me abrasan, si para ellos
}

11. Vers 524-525.

Toutes les citations sont tirées de l'édition de l'édition de Manuel Tudela déjà citée.

12. Silveira : La hermosa Dońa Isabel v. 1 Infanta de Portugal [...] 
Cette tirade est à prendre très au sérieux dans la mesure où aussi bien les forces telluriques qu'elle met en branle que l'écho de la présence d'Énée - évoqué comme avatar de la princesse Leonor -, dévirilisent don Juan, c'est-àdire lui font perdre le sens de lui-même, et signalent une puissance hors norme humaine de l'amour ici exprimé. Le départ d'un Énée féminin représente pour don Juan-Didon une perte affolante et dévastatrice qui est celle du principe même de vie et d'amour. Le renversement des principes masculin et féminin devient ici le signal d'une perte totale de contrôle de la part du personnage qui l'opère et la subit en même temps, une perte totale de pouvoir sur lui-même bien sûr, mais aussi et surtout une dépendance absolue vis-à-vis d'un amour passionnel échappant à toute rationalité humaine. Or, quelques scènes plus loin (scènes sur lesquelles nous allons revenir), don Juan se voit libéré de son mortel désespoir lorsque dońa Leonor, le découvrant triste et muet, l'enjoint de la suivre en Allemagne en qualité de gentilhomme :

$\begin{array}{ll}\text { Leonor: } & {[\ldots]} \\ & \text { Juntos nos hemos criado: } \\ & \text { lo que la niñez imprime } \\ & \text { nunca el tiempo lo ha borrado; } \\ & \text { ella da causa a que estime } \\ & \text { la fe que me habéis mostrado. } 485 \\ & \text { En mi nave os embarcad. }[. . .] \\ & \text { Don Juan, siempre os tuve amor } \\ & \text { Servidme en esta jornada }{ }^{14} .\end{array}$

Ces vers s'intercalent au cœur d'un échange entre Isabel et Leonor qui, loin de contredire ou de rendre plus diffuse l'interprétation que nous en ferons (et que don Juan, pour son plus grand malheur s'avère incapable de faire) participe de celle-ci :

$\begin{array}{ll}\text { Leonor [a Isabel]: } & \text { Mejor } \\ & \text { pena y lágrimas daré } \\ & \text { en empeños del amor } \\ & \text { que, desde niña, cobré } \\ & \text { a vuestra majestad }{ }^{15} .\end{array}$

Ainsi que nous pouvons le comprendre ici, si Leonor reçoit l'amour de don Juan c'est au nom de leur enfance, c'est-à-dire comme une sœur et non comme une amante, ce que le galant ne comprendra que plus tard au cours d'une scène des plus révélatrices. En dépit de toutes les évidences en effet, en dépit des avertissements de ses amis ${ }^{16}$ et de son serviteur et en dépit des remontrances

13. Vers 197-213.

14. AcVers 481-486 et 494-495.

15. Vers 497-501.

16. Fernando ¿Contra un águila imperial volais? No la alcanzaréis. 
implicites de Leonor elle-même, don Juan s'obstine à se faire aimer de la dame au point de commettre l'irréparable. De fait, Leonor, qui s'apprête à se rendre auprès de son époux, trébuche devant don Juan, lequel se précipite pour l'empêcher de chuter mais ne peut s'empêcher de se déclarer et s'attire en retour le plus cinglant des refus :

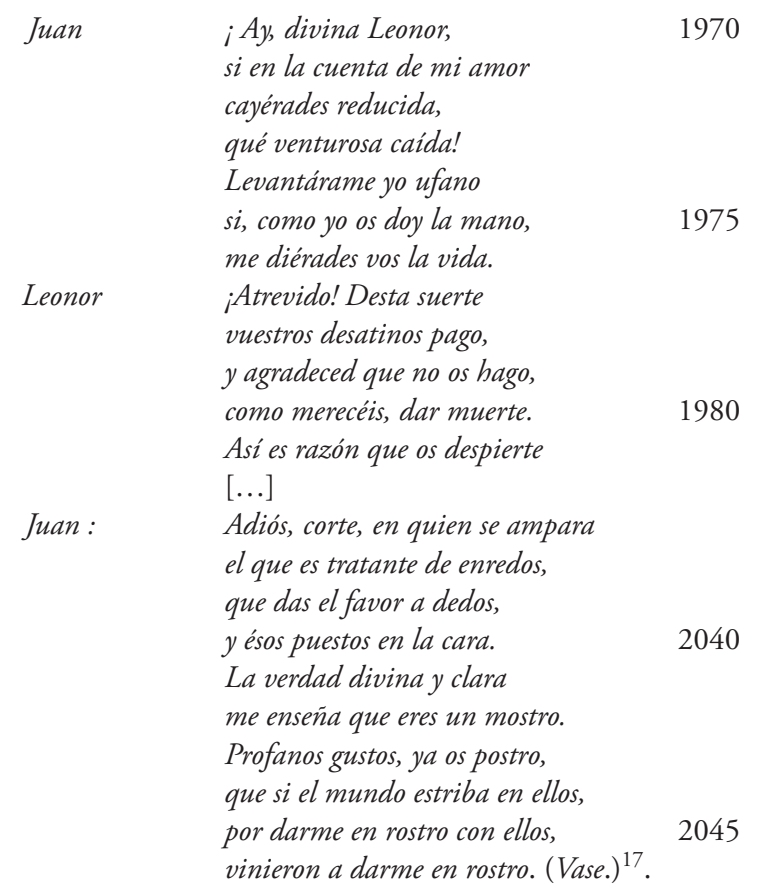

La désastreuse expérience vécue par Juan de Meneses et qui s'achève à la fin de l'acte deux, c'est-à-dire alors même que Beatriz est sur le point de subir le supplice infligé par la reine rendue folle de jalousie, est d'une importance essentielle dans l'économie générale de la pièce. De fait, elle nous présente, de leur point de vue, en vision subjective en quelque sorte, ce que vit chacun des galants présents dans l'œuvre - Juan II compris - en prise avec l'amour que leur inspire doña Beatriz. Certes, ils ne finiront pas, à l'instar de don Juan, par entrer

$\begin{array}{ll}\text { Juan } & \text { Es amor sacre sublime } \\ \text { empresa de su fuego es, } \\ \text { conde, o vencer o morir. } \\ \text { Venceréla o moriré. }\end{array}$

656

La présence de l'aigle, quand bien même s'agit-il d'une allusion à l'empereur d'Allemagne, n'est pas sans évoquer, pour peu qu'on le mette en relation avec le prénom Juan, l'aigle de Patmos de l'évangéliste. Or, comme nous l'apprenons à l'acte III par une didascalie (entre les v. 3051 et 3052) qui interrompt la révélation de Saint Antoine, don Juan est effectivement entré dans les ordres et s'adonne à la méditation et à l'écriture : "En lo alto de todo, entre una peñas estará don Juan de Meneses de fraile francisco, con una pluma en la mano, contemplando arriba en una imagen de la Concepción, y un libro abierto y blanco en la otra, en que parece que escribe, y una águila que con el pico le tiene el tintero".

17. Vers $1970-1981$ et 2037-2046 
dans les ordres, mais tous, et avant tout le roi Juan II, figure métonymique par excellence de tous les autres galants, subiront à leur échelle la même frénésie et la même souffrance que don Juan. Pour ne pas constituer la trame principale de la pièce, l'épisode concernant don Juan est une sorte de déclinaison spéculaire ${ }^{18}$ de l'argument central de la pièce : la difficulté à entrer dans le bon amour. Si les autres personnages masculins ne comprennent pas la nature de l'amour qui les aimante à Beatriz, don Juan de Meneses, lui, accède à la révélation de l'amour divin par l'évidence douloureuse de l'amour humain (entre un empereur et son impératrice). Cette douloureuse révélation lui permet d'accéder à une autre vérité, à savoir que l'amour qui l'habite et le meut, ce désir vers lequel il est entraîné n'est pas terrestre mais divin.

Outre le lien familial qui unit les deux personnages évoqués, un second élément établit une continuité entre le destin du frère et de la sœur, à savoir le prénom Juan ${ }^{19}$ qui est le même que celui du roi du Portugal, lequel est, en outre, "le second " (Don Juan Segundo). Dans l'un et l'autre cas, le prénom porté par les deux personnages d'amoureux « fous " est celui de l'apôtre préféré du Christ, Juan, celui là-même que le Christ "lègue $"^{20}$ à sa mère, alors qu'il agonise sur la croix, qu'il lui confie en lui demandant de le considérer comme son nouveau fils. Le fil subtil, discret et ininterrompu qui unit les personnages les uns aux autres passe indéniablement par ces deux Juan, le premier, frère de Beatriz et le second, son roi, deux figures masculines de pouvoir (familial, patriarcal, royal) subjuguées et affolées par un amour dont ils ne maîtrisent pas la puissance. Ce fil passe donc également par deux cours, celle de l'empereur d'Allemagne et celle du roi de Castille, inscrivant par là-même dans une forme d'universalité la leçon doctrinale et éthique de la pièce.

Si nous avons choisi comme porte d'entrée de l'analyse de la pièce l'histoire de don Juan de Meneses, laquelle nous a permis de jeter quelques bases conceptuelles de notre travail, il est temps à présent de nous tourner vers la trame principale de l'œuvre.

18. Manuel Tudela décrit ainsi la structure des deux premiers actes comme étant " circulaire " : El análisis de los dos primeros actos revela que su estructura es circular, en el sentido de que la acción secundaria rodea a la principal, p. 842.

19.Certes, les personnages de la pièce sont presque tous issus de l'histoire du Portugal et de la Castille et portent donc les noms restés à la postérité comme étant les leurs. L'on pourrait donc être tenté de renoncer à toute lecture poétique des noms des personnages dramatiques au nom, précisément, de l'implacable réalité historique, laquelle rendrait paradoxalement arbitraire - puisque contraint par l'état civil - le baptême de tel ou tel personnage. Il nous semble que ce serait faire peu de cas de l'intention et de la créativité du dramaturge à l'heure de choisir parmi les très nombreux épisodes et figures de l'histoire d'Espagne (qu'on nous pardonne cet anachronisme). Il y a fort à parier en effet que la richesse symbolique des noms réunis par l'épisode de la vie de Beatriz de Silva ici conté, à commencer par celui de l'héroïne elle-même, sur lequel nous reviendrons bientôt, joue un grand rôle dans le choix opéré par Tirso. En tout état de cause, les noms des personnages, pour authentiques qu'ils soient, n'en deviennent pas moins, une fois qu'ils sont intégrés à la fiction dramatique, des éléments à part entière de la poétique tirsienne et doivent donc être traités comme tels.

Nous rappellerons les mots de Roland Barthes qui dans "Critique et vérité ", écrit : « Les écrivains sont du côté de Cratyle, non d'Hermogène ». Barthes 1966: 62.

20. Dolto $1977: 63$. 
Dès le début de la pièce, Beatriz apparaît entre deux galants, l'un castillan, don Pedro Girón et l'autre portugais, don Pedro Pereira. Les festivités, liées aux épousailles et au départ de la future reine Isabel et de la future impératrice Leonor, battent son plein à Lisbonne et les deux aristocrates se disputent le droit d'accompagner dońa Beatriz. Si la galanterie empressée dont rivalisent les deux personnages peut apparaitre, au premier abord, comme relativement archétypale, très vite, l'insistance de l'un et de l'autre à prendre le dessus sur son opposant, la surenchère dans l'autosatisfaction aux limites du comique voire du ridicule à l'heure d'évaluer l'amabilité de la dame à leur égard (les deux galants commentent à leur avantage le fait d'être pour l'un à la droite de la dame et pour l'autre à sa gauche) et le commentaire final de Beatriz elle-même (Hidalgos, paso, /que me descuartizáis a cada paso) tout à la fois moqueur et agacé, font basculer la scène dans un autre type de démonstration. De fait, cette scène assez frénétique s'avère bien plus qu'une simple scène de séduction. Les deux galants se dressent l'un face à l'autre, tous ergots dehors, et semblent dépassés par la force de leur propre désir au point d'en venir presque à maltraiter l'objet de leurs attentions :

$\begin{array}{ll}\text { Girón: } & \text { Yo, que al izquierdo voy, no creo } \\ & \text { que pierdo } \\ & \text { la acción de venturoso, pues me cabe } \\ & \text { el corazón que, yendo al lado } \\ & \text { izquierdo, } \\ & \text { podré experimentar tierno y suave. } \\ & \text { Más noble es el derecho. } \\ \text { Pereira: } & \text { Si sois cuerdo, } \\ \text { Girón: } & \text { ved que del corazón gozo la llave. } \\ \text { Pereira: } & \text { Sabréosla yo quitar. } \\ \text { Beatriz: } & \text { Fidalgos, paso, } \\ & \text { :que me descuartizáis a cada paso } \\ & \end{array}$

L'aspect indéniablement comique de la situation, s'il met en relief l'esprit de Beatriz tout autant que son charme, n'enlève rien au fait que cette première apparition fixe un certain nombre de points caractéristiques de l'amour suscité par la dame. Tout d'abord, il est universel. En effet, avec un aristocrate castillan et un autre portugais, c'est métonymiquement toute la péninsule et donc tout le monde chrétien (si la pièce se passe dans les années 1440 elle a été écrite dans les années 1620) qui est concerné. Il est ensuite hyperbolique, au point que de brillants aristocrates en perdent le sens même de la mesure. Par ailleurs, et ce point est particulièrement important dans la perspective qui nous occupe ici, à aucun moment la dame ne semble vouloir chercher à susciter un tel attachement de la part des galants. Autrement dit, Beatriz n'est ni une coquette en mal de séduction ni ce qu'on appellerait aujourd'hui une manipulatrice narcissique. Enfin, il nous faut nous arrêter ici sur les noms des deux galants. Tous deux sont des " Pedro" (au reste Beatriz souligne elle-même 
cette coïncidence : Dos Pedros a mi lado, ilustres llevo,/cada uno galán, noble, valiente, sin saber [...]/distinguir lo que va de Pedro a Pedro ${ }^{22}$ ) l'un Girón, l'autre Pereira. Si nous retrouvons le chemin des Évangiles avec la double présence de Pierre, l'apôtre fondateur de l'église chrétienne, le fidèle malgré ses humaines faiblesses, ce sont surtout les patronymes qui vont ici nous intéresser ${ }^{23}$. De fait, le nom Girón, homonyme du lambeau d'étoffe représentant l'hymen immaculé (jirón), apparaît une fois dans la pièce en tant que substantif, au début de l'acte trois, lorsque la Vierge, sous la forme d'une fillette de bleu et de blanc vêtue, apparaît à Beatriz enfermée dans l'armoire :

$$
\begin{aligned}
& \text { [...] } \\
& \text { Yo soy la privilegiada } \\
& \text { cuya cándida creación, } \\
& \text { hecha por Dios ab initio, } \\
& \text { para su Madre eligió, } \\
& \text { que, habiéndose de vestir } \\
& \text { la tela que tejió amor, } \\
& \text { quiso preservar sin mancha } \\
& \text { en mí limpio este jirón } \\
& \text { al poner el pie en el mundo } \\
& \text { donde el hombre tropezzít. } \\
& {[\ldots]}
\end{aligned}
$$

Plus encore, peut-être, que l'Immaculée Conception de la Vierge, ce que révèle le discours de la fillette tient à la notion d'amour et à l'imperfection humaine, laquelle confond trop souvent concupiscence et amour. Ce jirón inscrit également la présence mariale au cœur du texte. Il installe donc, comme en arrière-plan du drame, la présence du couple Marie-Christ, lequel s'incarne en Beatriz elle-même. L'apparition de l'Enfant-Vierge, matérialisation de la virginité littérale, vient au demeurant en contrepoint à la présence relevée plus haut d'une Didon suicidaire et donc impie mais aussi au destin très humain des damas de l'histoire ici contée.

Quant au second Pedro de la pièce, dont le nom Pereira désigne, en portugais, le poirier, il met en garde contre les dangers d'un amour incompris. Le poirier,

22. Vers 313-316.

23. Suivant en cela les enseignements de Maurice Molho, nous considérons qu'aucun signifiant et moins encore un signifiant onomastique, ne peut être arbitraire dans une œuvre littéraire. Maurice Molho explique en effet que « la nomination du personnage n'est rien d'autre, au vrai, qu'un acte d'onomatomancie (...). Le personnage, ainsi que la personne, est donc tout entier dans son nom. Le nom énonce, en effet, un programme de comportement et d'actes que le lecteur, habile onomatomancien, est sollicité de décrypter dans le jeu dont l'artiste lui livre d'avance la clé. » Molho 1984 : 88-89.

De la même manière, M. Molho indique dans son introduction au Persille : " [...] le nom est le signifiant de la personne, ce qui permet de la connaître et reconnaître par le jeu des traits ou éléments qui se combinent pour former une onomastique particulière, nécessairement transcendante à l'individu qui s'y identifie ", Molho $1994: 41$.

24. Vers 2127-2136. 
en effet, est au centre d'une très célèbre histoire de désir et d'envie racontée par Saint Augustin dans ses Confessions:

Il y avait un poirier, près de la vigne de mon père, dont les poires n'étaient ni fort belles à la vue, ni fort délicieuses au goût. Nous nous en allâmes, une troupe de méchants enfants après avoir joué ensemble jusqu’à minuit, comme ce désordre n’est que trop commun : nous nous en allâmes, dis-je, secouer cet arbre pour emporter tout ce qu'il avait de fruits. Et nous nous en revînmes tout chargés de poires, non pour les manger, mais seulement pour les prendre, quand on les eût dû jeter aux pourceaux (quoique nous en mangeâmes quelque peu) nous contentant du plaisir que nous trouvions à faire ce qui nous était défendu ${ }^{25}$.

Certes, doña Beatriz est d'une indéniable beauté (aux limites du surnaturel pourrions-nous même dire) mais le danger qui menace les galants est précisément celui explicité par la confession d'Augustin, celui d'un simple désir de possession détaché de tout besoin ou de tout amour, lequel s'inscrit également dans la tentation du fruit défendu telle que l'évoque Augustin dans ce souvenir de jeunesse.

Cette première apparition de dońa Beatriz dans la pièce fixe, ainsi que nous pouvons le constater, un certain nombre d'éléments clefs, lesquels se mêlent pour constituer une sorte de maelström qui emporte les personnages hors des limites du raisonnable telles que devrait les fixer leur statut socio-dramatique. Le fait est que les deux éléments catalyseurs, amour et pouvoir, sont explicitement présents dans le texte lui-même lorsque don Fernando, l'ami de don Juan s'exclame :

$\begin{array}{ll}\text { Fernando } & \text { En noche triste nuestro reino quede, } \\ & \text { pues se le ausentan juntos tres Apolos. } \\ \text { Beatriz } & \text { Ese titulo sólo se concede } \\ & \text { a las infantas, conde de Arroyolos, } \\ & \text { que en mi no caben excelencias tantas. } \\ \text { Fernando } & \text { Reina en belleza sois, si ellas infantas }{ }^{26} .\end{array}$

Le renversement de l'attribution des titres sur la base d'un nouveau critère, la beauté et non plus le lignage, n'est pas à considérer comme une simple hyperbole célébrant la beauté de Beatriz, mais bien comme un indice fort quant à la véritable nature dramatique du personnage dont l'aura se propage sans limite de temps ni d'espace (la référence mythologique, pour archétypale qu'elle puisse paraître, est également une extraordinaire ouverture spatiotemporelle) et encore moins d'humaines conventions. Dans le même ordre d'idée, la noche triste, qu'évoque don Fernando nous semble faire écho inversé à une autre nuit, beaucoup plus heureuse celle-là, celle de San Juan de la Cruz qui, dans son poème En una noche oscura, écrit - entre autres - cette strophe :

En la noche dichosa

en secreto que nadie me veía

25. Augustin $1993: 74$.

26. Vers 335-340. 


$$
\begin{aligned}
& \text { ni yo miraba cosa } \\
& \text { sin otra luz y guía } \\
& \text { sino la que en el corazón ardía. }
\end{aligned}
$$

Don Fernando, comme tous les autres personnages masculins de la pièce au reste, est prisonnier d'une nuit non encore illuminée par la lumière mystique évoquée par Jean de la Croix. Ce qu'exprime le galant dans son compliment hyperbolique - et ce à son corps défendant - apparait à cette aune comme bien plus profond et essentiel que la perte pour Lisbonne d'une beauté de plus. La prééminence donnée à Beatriz sur les altesses en titre au nom de sa beautée ${ }^{27}$, la présence de la nuit dans le discours de don Fernando, sont les premiers éléments manifestes de la mise en question du pouvoir terrestre que s'attache à opérer la pièce et que vont développer puissamment toutes les scènes convoquant le roi Juan II et la reine Isabel ainsi que nous allons le voir à présent.

Ainsi que nous l'avons indiqué à deux reprises, un malheureux échange de portraits induit le roi en erreur et lui fait prendre doña Beatriz pour doña Isabel, allumant chez lui un amour irrépressible :

Rey $\quad$ (A él aparte.) iAy, don Pedro! ¿Qué habéis hecho?
(Aparte.) Aposentóse en mi pecho
doña Beatriz, que sosiega
de mi amor la llama ciega,
y a Isabel dejo burlada,
que el alma como es posada
se da al primero que llega.
$\left[\right.$ Alto.] Venga vuestra majestad ${ }^{28}$.
$[\ldots]$

Cet amour possède le roi sans qu'il y puisse rien faire mais ici encore, nous sommes loin du stéréotype du souverain indigne épris d'une dame inférieure et prêt à toutes les bassesses, pour parvenir à ses fins ${ }^{29}$. L'amour du roi est bien moins banal, comme le prouve cet aveu :

$$
\begin{aligned}
& \text { Yo os adoro, Silva bella; } \\
& \text { fácil en el alma entrastes, } \\
& \text { tras vos la puerta cerrastes; } \\
& \text { mal os echará por ella } \\
& \text { de la reina la hermosura, }
\end{aligned}
$$

27. Il semble également difficile de ne pas entendre, dans l'insistante référence à la beauté de Beatriz, les échos d'une pensée néoplatonicienne faisant du beau le marqueur par excellence du bien et du vrai.

28. Vers 995-1002.

29. Le roi, conscient du caractère inconvenant et inouï de son amour pour Beatriz espère que la campagne qu'il s'apprête à mener contre les maures de Grenade va le guérir de cette passion : deseos amorosos e inconstantes/que hacéis que os peche el alma y pague censo; si la paz hace guerra a los amantes/mi paz con esta guerra recompenso. :jDichoso si con ella divertidolapago incendios y a Beatriz olvido! Vers 1584-1589. 


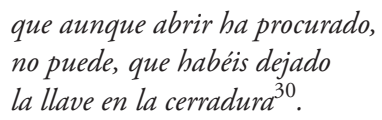

Et plus loin ces quelques vers adressés à doña Inés, amie de Beatriz qui feint de croire que le roi pense à dońa Isabel :

No, doña Inés, que aunque reina

en el alma que adoralla

jura, puede ser vasalla

de quien me abrasa la reina ${ }^{31}$.

[...]

Avant de procéder à l'analyse de ces vers, il nous semble important de nous arrêter sur le personnage de Beatriz elle-même et sur son attitude à la cour. En effet, si la dame est consciente de l'emprise qu'elle exerce sur les hommes, à aucun moment, nous l'avons déjà dit, elle ne cherche à la susciter. A dońa Inés qui lui reproche amèrement de ne laisser aucun galant aux autres dames de la cour, et en particulier à elle, Beatriz réplique :

$\begin{array}{cc}\text { Todo me agrada } \\ \text { Beatriz: } & \text { Ya todos quiero igualar, } \\ & \text { Y no me enamora nada. } \\ & {[\ldots]} \\ \text { Inés : } & \text { En fin, ¿ha de dar favores } \\ & \text { A todo amante tu agrado? } \\ \text { Beatriz: } & \text { ¿Qué quieres ? Guantes y flores, } \\ & \text { Danlos las tiendas y el prado : } \\ & \text { No he de ser yo menos que ellos }\end{array}$

Une lecture superficielle de ces vers pourrait laisser penser que Beatriz fait ici montre d'une insensibilité condamnable et d'un narcissisme coupable. Il en va de même, lorsque, à dońa Inés qui la supplie de ne pas jeter son dévolu sur don Pedro Girón dont elle est éprise, Beatriz répond d'abord :

Todo lo que es cosa ajena

Engendra apetito en mí.

En viendo en otra gala,

Luego por ella me muero

Hasta estar de envidia mala;

Al que desdeñaba, quiero

Si otra dama le regala.

Mira tú de qué manera

Sufrirá mi inclinación

Que lo que quieres no quiera ${ }^{33}$.

\footnotetext{
30. 1234-1241

31. Vers 1254-1256

32. Vers $1029-1031$ et 1068-1072.

33. Vers 1095-1104.
} 
Or, ces déclarations sont avant toute chose les dernières manifestations d'une faiblesse humaine que non seulement Beatriz finira d'expier durant ses trois jours d'enfermement dans l'armoire où l'a emprisonnée la reine mais qu'elle renie à peine les a-t-elle prononcées :

\section{Ahora bien, yo te prometo, \\ Doña Inés, hacerlo asi; \\ $Y$ sólo por tu respeto, \\ Olvidarle desde aqui ${ }^{34}$.}

Quand bien même elle cèdera à l'insistance de don Pedro Girón et lui remettra un trophée (son cure-dent) c'est avant tout pour ne pas priver le palais du bonheur de la présence du galant - qui menace de ne pas s'y rendre - au tournoi organisé par le roi. Il s'agit donc bien ici d'un premier sacrifice et non d'une coquetterie de dame volage ${ }^{35}$.

Il nous semble décisif ici de considérer les paroles de doña Beatriz dans leur plus stricte littéralité et en particulier lorsqu' elle déclare $Y$ a todos quiero igualar. De fait, il n'est nullement question pour la dame de se jouer de l'amour des galants qui la sollicitent pas plus qu'il n'est question de le rejeter. La tension qui s'installe ici entre les déclarations de la dame et la réception de ces déclarations par Doña Inès, qui va se considérer trahie lorsqu'elle assiste à la rencontre entre Pedro Girón et Beatriz, est celle liée à l'incompréhension d'une relation à l'amour absolument inédite et que Beatriz devra presque payer de sa vie avant qu'elle ne soit comprise par les représentants du pouvoir que sont le roi Juan II et la reine Isabel.

Pour en revenir aux aveux du roi - faits d'abord en aparté puis à doña Inés - ils vont s'accompagner, malgré son désir sincère de mettre un terme à sa passion $^{36}$, d'un remarquable dévoiement du pouvoir royal le plus symbolique qui soit : le mariage. Après que Inés - cherchant ainsi à se venger de ce qu'elle considère comme la trahison de Beatriz - a révélé à la reine Isabel la demande inconvenante du roi, la reine met son époux à l'épreuve en lui demandant de marier Beatriz à l'un des galants qui la courtisent afin de mettre fin aux souffrances de tous les autres et de ramener le calme au palais ${ }^{37}$. Chacun des prétendants est récusé par le roi pour des motifs plus fallacieux les uns que les autres et le dialogue, de plus en plus tendu, s'achève par une violente fin de non-recevoir infligée par don Juan II à doña Isabel :

34. Vers 1112-1115.

35. No quiera Dios que por mílpierda el Palacio su fiesta;/volved, no os partáis asil, que si tan caro me cuestalcumplir lo que prometíl, por mejor tengo agradaros/que triste el Palacio esté./[...] Acte II, v. 1172-1178.

36. Note 28 p. 15.

37. La reine a en effet pu constater lors d'une audience donnée aux Grands de la Cour que tous souhaitaient ardemment épouser Beatriz sans qu'il soit possible de déterminer si l'un d'eux en était particulièrement aimé. 
No me canséis, señora,

Que no gusto se case por agora. (Vase.) $)^{38}$.

Outre le fait que ce refus confirme les soupçons de la reine et précipite son intention homicide, il relève également, comme nous le disions, de l'abus de pouvoir (ou de son déni) dans la mesure où le roi se refuse à accomplir ce qui, dans la structure globale qu'est la comedia, relève précisément de son seul pouvoir, et en constitue un élément structurant. L'amour insensé provoqué chez Juan II par Beatriz, atteint donc symboliquement les fondements même de la fonction royale. Il en va évidemment de même pour la reine, laquelle, toute portugaise - et donc amoureuse redoutable ${ }^{39}$ - qu'elle soit en vient à des extrémités qui non seulement terrifient doña Inés, dans un évident effet de théâtre dans le théâtre ${ }^{40}$, mais qui relèvent de la pure démence criminelle :

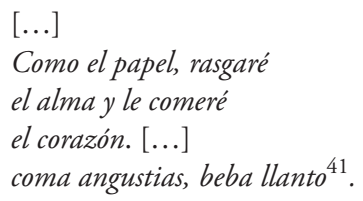

Nous sommes ici au paroxysme d'une crise se déroulant sur tous les fronts, touchant tous les personnages de la pièce et dont l'élément catalyseur est, plus que la seule beauté de doña Beatriz, l'amour qu'elle suscite quasiment à son corps défendant. Certes, la dame va être miraculeusement sauvée par la Vierge, mais ce sauvetage ne prend vraiment sens, du point de vue de l'économie globale de l'œuvre, que si l'on veut bien considérer Beatriz pour autre chose que la seule émanation d'une personnalité historique créatrice d'un ordre marial lié au pouvoir royal. Ce sera là l'objet de la dernière partie de notre travail.

C'est à nouveau au nom que nous allons nous intéresser car il constitue une sorte de clef de voûte de l'édifice poétique qu'est cette pièce. Beatriz (dont le prénom anticipe la sainteté, ou tout du moins, la béatitude) porte le nom de Silva, opportunément convoqué par le roi dans un vers cité précédemment : «Yo os adoro Silva bella». Or ce nom, forme latinisante du mot selva, désigne, comme l'indique le Dictionnaire des Autorités, un "Lugar lleno de árboles, malezas y matas que le hacen naturalmente frondoso ".

La convocation littérale de ce lieu très particulier associée au caractère insaisissable de Beatriz, qui se laisse aimer de tous sans pour autant se laisser capturer par l'amour d'autrui, installe dans le texte un arrière-plan poétique très subtil et très puissant en même temps qui est celui du Cántico espiritual de San Juan de la Cruz (déjà évoqué pour sa Noche oscura), que Tirso a pu lire

38. Vers 1636-1637.

39. Il est en effet important de garder à l'esprit que la reine Isabel est parfaitement éprise de son époux : Quiéroos mucho, estoy celosa déclare-t-elle vers 2614.

40. La dame s'exclame ainsi lorsqu'elle comprend l'intention de la reine : ;Gran señora... ! Puis elle tente de la raisonner : Después/ que aquesta tempestad fiera/pase, abrirla mandarás ;/ que es castigo riguroso. Pour enfin s'écrier : No jures, señora, más. Vers 1792, 1808-1810, 1812.

41. Vers $1750-1752$ et vers 1816. 
sous forme de manuscrit ${ }^{42}$. Immédiatement après avoir prononcé le vers que nous venons de citer, le roi poursuit en effet en constatant : "fácil en el alma entrastes». Si ce vers tient bien évidement du topique amoureux, il n'en reste pas moins que la proximité du signifiant alma avec les références à la silvalselva, à l'amour puissant et spontané et à l'errance amoureuse installe dans le texte un paradigme subtil qui fait affleurer le souvenir du Cántico et en particulier, semble-t-il, celui des quatre premières chansons :

¿Adónde te escondiste,
Amado, y me dejaste con gemido?
Como el ciervo huiste,
habiéndome herido;
sali tras ti clamando, y eras ido.
Pastores, los que fuerdes
alla por las majadas al otero:
si por ventura vierdes
aquel que yo más quiero,
decidle que adolezco, peno y muero.
Buscando mis amores
iré por esos montes y riberas;
ni cogeré las flores,
ni temeré las fieras,
y pasaré los fuertes y fronteras.
¡Oh bosques y espesuras,
plantadas por la mano del Amado!
¡Oh prado de verduras,
de flores esmaltado!
Decid si por vosotros ha pasado

Beatriz pourrait bien être ce ciervo que tous poursuivent avec une frénésie inaccoutumée et qui blesse d'une blessure d'amour, à la douleur incompréhensible, chacun de ceux qui l'approchent. Beatriz de Silva devient ainsi une véritable figure christique suscitant une forme d'amour inouï puisque divin et incompris de ceux qui l'éprouvent (et de ceux qui le subissent comme la reine Isabel). Cet amour émanant de la dame, dans la mesure où il est divin, est également sacrificiel. C'est le sens à donner au calvaire de trois jours enduré par Beatriz dans le plus grand silence afin de ne pas porter tort à la reine :

42. Pour la diffusion manuscrite du Cántico espiritual voir : San Juan de la Cruz, Cántico espiritual, ed. de Eulogio Pacho, Madrid, Fundación Universitaria espańola, 1981, Chapitre II, «En el ambiente religioso espańol», p. 106-117 et, pour les canciones citées, p. 586-589. Nous tenons à remercier Nadine Ly pour cette précieuse indication bibliographique.

43. Cántico espiritual, ibid., canciones 1 à 4. 


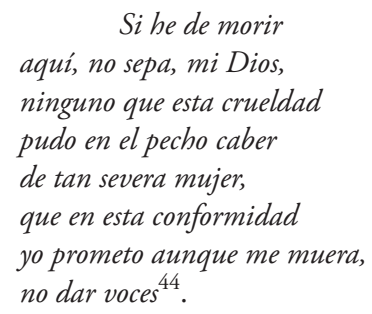

L'ensevelissement de la dame pendant trois jours n'est bien sûr pas sans rappeler les trois jours passés par le Christ au tombeau avant sa résurrection ${ }^{45}$. L'intervention de la Vierge sauve Beatriz de la mort et permet à la cour d'assister à une nouvelle forme de résurrection, trois jours après la disparition de la dame. Beatriz évoque d'ailleurs clairement cette disparition dans la lettre d'explications qu'elle laisse avant de fuir le palais ${ }^{46}$. Sa résurrection, à l'instar de celle du Christ se sacrifiant pour racheter les péchés des hommes, permet au roi et à la reine de faire amende honorable et de réintégrer leur fonction véritable. $\mathrm{Ni}$ le dévoiement coupable du roi, ni les excès mortifères de la reine ne sont punis dans la pièce : dans un cas comme dans l'autre, ils ont été commis par amour et la pièce nous rappelle que, dans une perspective chrétienne, rien de ce qui est fait par amour ne peut être considéré comme mauvais. Autrement dit, non seulement Beatriz par un sacrifice évité, apprend aux personnages gravitant autour d'elle la véritable valeur de l'amour divin, mais elle déplace également, ou plus exactement elle replace, les notions de pouvoir et d'autorité dans la perspective de l'amour divin conçu comme caritas. Doña Beatriz et l'amour qu'elle suscite autour d'elle permettent de dissocier amour ou charité et désir ou concupiscence ainsi que désir et besoin mais permettent également d'apprendre à aimer. Le pouvoir royal, arbitraire, abusif et violent lorsqu'il est soumis à la loi du désir, recouvre sa légitimité et sa force lorsqu'il se plie à l'autorité de l'amour divin, lequel, nous l'avons vu, en particulier avec l'exemple de don Juan de Meneses, n'est pas exempt de souffrance. La présence d'Álvaro de Luna dans la pièce, dont nous n'avons pas du tout traité jusqu'ici, est à lire de la même manière. De fait, si tous les Grands de la Cour reprochent amèrement au roi Juan II de s'être littéralement laissé marier par son conseiller, aussi bien ce dernier que le roi justifient cette extraordinaire initiative par l'amitié profonde qui les unit. Or cette amitié hautement valorisée par le texte tirsien qui jamais ne la met en doute ni ne la condamne, n'est pas ici non plus exempt de souffrance

44. Vers 1799-1805.

45. Luc raconte dans son évangile que deux hommes en habit éblouissant déclarent aux saintes femmes venues laver le corps de Jésus dans son tombeau: "Pourquoi cherchez-vous le Vivant parmi les morts ? Il n'est pas ici ; mais il est ressuscité. Rappelez-vous comment il vous a parlé quand il était encore en Galilée : Il faut, disait-il, que le Fils de l'homme soit livré aux mains de pécheurs, qu'il soit crucifié et qu'il ressucite le troisième jour ». Lc 7245.

46. Sospechas de Vuestra Alteza y desengaños míos (en tres días que estuve sepultada) me enseñaron los peligros de Palacio, pues al cabo de ellos podré afirmar que resucité el tercero día. [...] entre les vers 2738 et 2739 . 
ni de sacrifice. Álvaro de Luna sera promptement condamné à mort par le roi lui-même et chacun des deux acceptera ce sacrifice terrible, consenti par l'un comme par l'autre, pour le bien du royaume.

Pour conclure cette analyse de la pièce Doña Beatriz de Silva, il nous parait important de souligner combien une structure dramatique souvent perçue comme relativement hétérogène s'avère au contraire extraordinairement structurée et cohérente. La pluralité apparente des thématiques présentes dans la pièce (palatines, historiques, hagiographiques, comme nous le soulignions en introduction) n'a en réalité d'autre but que de se mettre au service de la visée universelle (sens du mot catholique) de l'œuvre. Au-delà de l'alliance du Pouvoir royal et de l'Église indéniablement célébré par le théâtre du XVII siècle et par Tirso lui-même ${ }^{47}$, il s'agit pour le mercédaire de rappeler que tout pouvoir, pour être légitime, doit se soumettre à une autorité transcendante laquelle ne peut être autre que celle de l'Amour du Christ. La pièce dramatise donc avant toute autre chose la difficulté pour chaque croyant à recevoir et à comprendre l'amour divin et la nécessité pour la société chrétienne d'accepter de passer du pouvoir temporel à l'autorité de l'amour divin à travers la personne même du roi.

Elle propose une leçon de vie dépassant amplement les simples contingences socio-historiques (qu'elle englobe) puisque chacun des personnages se trouve, dans son expérience dramatique, confronté à l'amour. S'il excuse tous les comportements, s'il pardonne tous les excès commis en son nom et sous son emprise, l'amour peut être source de souffrance et de sacrifice dès lors qu'il se veut juste. Le pouvoir royal doit donc s'évaluer à l'aune de l'autorité supérieure et transcendante de l'amour du Christ pour les hommes et en adopter les principes fondamentaux s'il prétend être un pouvoir légitime et juste.

\section{Bibliographie}

Augustin, Les Confessions, Paris, Gallimard, collection Folio Classique, 1993.

Barthes Rolland, "Critique et vérité », Tel Quel, Paris, Seuil, 1966.

La Bible de Jerusalem, Paris, ed. Desclee de Brouwer, 1975.

Cervantès Miguel de, Les travaux de Persille et Sigismonde, Traduit et présenté par Maurice Molho, Paris, José Corti, 1994.

Dolto Françoise, L'évangile au risque de la psychanalyse 1, Paris, Éditions du Seuil, Collection Point, 1977.

Florit Francisco, "Ad devotionem excitandam: Dońa Beatriz de Silva de Tirso de

Molina ", Estudios Románicos, Volumen 16-17, 2007-2008.

47. José Antonio Maravall (entre autres) démontre avec pertinence la fonction propagandistique du théâtre baroque : [...] los medios de socialización que se dirigen a una masa para hacerla participar de tal imagen social, tienen, en su función integradora, un carácter conservador. Se persigue difundir y consolidar la imagen de la sociedad, establecida en apoyo de un sistema de intererses, con la pretensión de conservar su orden. Maravall 1975 : 266. 
Maravall José Antonio, La cultura del Barroco, Madrid, Ariel, 1975.

Maurel Serge, L'univers dramatique de Tirso de Molina, Publications de l'Université de Poitiers, 1971.

Molho Maurice, "Le nom, le personnage ", Le personnage en question, IVe colloque du S.E.L, Université de Toulouse-Le Mirail, 1984.

Oteiza Blanca, ¿Conocemos los textos verdaderos de Tirso de Molina ? in Varia lección de Tirso de Molina: actas del VIII Seminario del Centro para la Edición de Clásicos Españoles (Madrid, 5-6 de Julio de 1999) / edición de I. Arellano y B. Oteiza.

Palomo Vázquez, María del Pilar, La creación dramática de Tirso de Molina (II) http:// www.ucm.es/OTROS/especulo/numero8/palomo2.htm

San Juan de la Cruz, Cántico espiritual, ed. de Eulogio Pacho, Madrid, Fundación Universitaria española, 1981.

Tirso de Molina, Obras completas, Cuarta parte de comedias, I, Vol. 5, ed. crítica del I.E.T., dirigida por I. Arellano, 1999, p. 833-989 et Variantes, p. 1137-1152.

Vázquez Luis, Varia lección de Tirso de Molina : actas del VIII Seminario del Centro para la Edición de Clásicos Españoles (Madrid, 5-6 de Julio de 1999) / edición de I. Arellano y B. Oteiza. 
